\title{
Differential Effects of 'Vaping' on Lipid and Glucose Profiles and Liver Metabolic Markers in Obese Versus Non-obese Mice
}

\author{
Hui Chen ${ }^{1 \dagger}$, Gerard $\mathrm{Li}^{1+}$, Yik Lung Chan ${ }^{1}$, Hui Emma Zhang ${ }^{2}$, Mark D. Gorrell ${ }^{2}$, \\ Carol A. Pollock ${ }^{3}$, Sonia Saad ${ }^{3}$ and Brian G. Oliver ${ }^{1,4 *}$
}

${ }^{1}$ School of Life Sciences, Faculty of Science, University of Technology Sydney, Sydney, NSW, Australia, ${ }^{2}$ Centenary Institute, Faculty of Medicine and Health, The University of Sydney, Sydney, NSW, Australia, ${ }^{3}$ Renal Research Laboratory, Kolling Institute of Medical Research, The University of Sydney, Sydney, NSW, Australia, ${ }^{4}$ Respiratory Cellular and Molecular Biology, Woolcock Institute of Medical Research, The University of Sydney, Sydney, NSW, Australia

OPEN ACCESS

Edited by: Jorge Simon, CIC bioGUNE, Spain

Reviewed by:

Analia S. Loria,

University Press of Kentucky,

United States

Renata Guerra Sa,

Universidade Federal de Ouro Preto,

Brazil

${ }^{*}$ Correspondence:

Brian G. Oliver

brian.oliver@uts.edu.au

tThese authors have contributed equally to this work

Specialty section:

This article was submitted to Metabolic Physiology, a section of the journal Frontiers in Physiology

Received: 08 August 2021 Accepted: 18 October 2021 Published: 04 November 2021

Citation: Chen H, Li G, Chan YL, Zhang HE, Gorrell MD, Pollock CA, Saad S and Oliver BG (2021) Differential Effects of

'Vaping' on Lipid and Glucose Profiles and Liver Metabolic Markers in Obese Versus Non-obese Mice.

Front. Physiol. 12:755124.

doi: 10.3389/fphys.2021.755124
Tobacco smoking increases the risk of metabolic disorders due to the combination of harmful chemicals, whereas pure nicotine can improve glucose tolerance. E-cigarette vapour contains nicotine and some of the harmful chemicals found in cigarette smoke at lower levels. To investigate how e-vapour affects metabolic profiles, male Balb/c mice were exposed to a high-fat diet (HFD, $43 \%$ fat, $20 \mathrm{~kJ} / \mathrm{g}$ ) for 16 weeks, and e-vapour in the last 6 weeks. HFD alone doubled fat mass and caused dyslipidaemia and glucose intolerance. E-vapour reduced fat mass in HFD-fed mice; only nicotine-containing e-vapour improved glucose tolerance. In chow-fed mice, e-vapour increased lipid content in both blood and liver. Changes in liver metabolic markers may be adaptive responses rather than causal. Future studies can investigate how e-vapour differentially affects metabolic profiles with different diets.

Keywords: liver, abdominal obesity, free fatty acid, triglycerides, glucose tolerance

\section{INTRODUCTION}

In recent years, e-cigarettes have gained significant popularity among younger people, where they are considered as a must-have electronic and a 'safe cigarette'. Although e-cigarettes were originally marketed as a smoking cessation aid to reduce tobacco use, they are largely used recreationally by those who never used tobacco cigarettes, despite the latest reports of acute lung injury and death in the United States (Layden et al., 2019).

The negative impacts of tobacco smoking on metabolic disorders in adults and adolescents have been well documented (Oh et al., 2005; Weitzman et al., 2005). Nicotine alone (i.e. as a free chemical) can positively increase glucose uptake into tissues after chronic administration in both lean and obese rodents (Liu et al., 2003; Vu et al., 2014). The effects of tobacco smoke and pure nicotine can be different, as tobacco smoke contains more than 5,000 chemicals resulting in toxicity to the body, including increased risk of metabolic diseases and cancer. Nicotine, although addictive, is also anti-inflammatory (Kalra et al., 2004; Cohen et al., 2007). This leads to the discrepancies between in vivo studies using pure nicotine and direct cigarette smoke. E-cigarette vapour, which is claimed to be water and nicotine, is clearly not so innoxious 
as it contains various harmful chemicals which are also found in cigarettes (Li et al., 2021) that may potentially affect lipid and glucose metabolism. Due to the relatively recent emergence of e-cigarettes, there is limited research on the impact of e-cigarette use beyond the respiratory system, which is also dominated by studies funded by the tobacco industry (Pisinger and Døssing, 2014). Human studies in this area are difficult and are affected by various confounders, such as dietary habits, pre-existing health conditions and the dual use of e-cigarettes and tobacco cigarettes (Kim et al., 2020). E-cigarette users have been reported to have a larger body mass index, and a large proportion of dual users have abdominal obesity (Lanza et al., 2017; Górna et al., 2020; Kim et al., 2020). Thus, it is not surprising to find a higher risk of metabolic syndromes in this population, such as an increased blood glucose level or a prediabetic status (Lanza et al., 2017; Górna et al., 2020; Kim et al., 2020). Long-term longitudinal studies on e-cigarette users are not yet available because of their relatively recent entry into the market. Therefore, it is unclear whether the metabolic disorders in e-cigarette users are due to a natural progression due to pre-existing conditions/unhealthy lifestyles, e-cigarette use or the combination of both.

Obesity causes low grade systemic inflammation, due to the recruitment and accumulation of tissue macrophages in response to lipid influx (Lumeng et al., 2008). This is believed to be the dominant mechanism for the development of insulin resistance and liver steatosis during obesity. In the $\mathrm{ApoE}^{-/-}$ mouse, in which liver steatosis readily develops, the combination of e-cigarette aerosol and high-fat diet (HFD, 60\% fat) increased liver triglyceride deposition, oxidative stress, and DNA damage (Espinoza-Derout et al., 2019; Hasan et al., 2019). This was suggested to be due to increased lipolysis in the fat tissue, which increases plasma free fatty acid (FFA) levels, resulting in increased ectopic lipid synthesis and deposition in the liver (Espinoza-Derout et al., 2019). However, the dose of nicotine in this study was very high (equivalent to heavy smokers, i.e. 18 cigarettes/day). Additionally, conclusions from genetically modified mice may only apply to those with severe, pre-existing metabolic disorders. A recent review indicated that the evidence is inconclusive on the risk of type 2 diabetes in e-cigarette users and that current animal studies cannot provide an answer, either due to issues with experimental design (e.g. the use of non-vaporised e-fluids or pure nicotine) or the difficulties of eliminating confounders (e.g. duel use of tobacco cigarette and e-cigarette or pre-existing conditions prior to the use of e-cigarette; Górna et al., 2020). Therefore, a well-controlled animal study using wild-type rodent strains is necessary.

The manufacture and sale of e-fluids are not well regulated. For example, in New York, only the sale of tobacco-flavoured e-fluids is permitted to reduce the potential harm induced by

Abbreviations: ATGL, adipose triglyceride lipase; AUC, area under the curve of glucose change during the intraperitoneal glucose tolerance test; $\mathrm{CPT}$, carnitine palmitoyltransferase; FASN, fatty acid synthase; FOXO1, Forkhead box protein O1; HFD, high-fat diet; IPGTT, intraperitoneal glucose tolerance test; NEFA, non-esterified fatty acids; ORO, oil red O; Glut, glucose transporter; PEPCK, phosphoenolpyruvate carboxykinase; PPAR, peroxisome proliferator-activated receptor; SEM, standard error of the mean. the flavouring chemicals. However, in Australia, the sale of e-fluid is permitted without restrictions on flavour, but nicotine is not a legally allowed additive. While nicotine is the accepted addictive substance in the e-fluid, other additives (e.g. pyrazines) have been found in nicotine-free e-fluid, which alone can cause addiction (Alpert et al., 2015). We previously also found that exposure to nicotine-free e-cigarette vapour can increase lung inflammatory responses and impair memory in mice (Chen et al., 2018a, 2020). However, its impact on glucose and lipid metabolism is unclear. Therefore, using our well-established HFD diet feeding protocol (Glastras et al., 2016; Chen et al., 2018b; Komalla et al., 2020), we aimed to investigate in a mouse model whether nicotine-containing or nicotine-free e-vapour inhalation interacts with diet to affect glucose tolerance, blood lipid levels and liver metabolic markers.

\section{MATERIALS AND METHODS}

\section{Animal Experiments}

The experiments were approved by the Animal Ethics and Care Committee at Northern Sydney Local Health District (RESP17/93) and all experiments were performed according to the Australian National Health \& Medical Research Council Guide for the Care and Use of Laboratory Animals. Balb/c mice were used due to their susceptibility to cigarette smokeinduced lung pathology and systemic inflammatory and fibrotic responses, as well as our previously published protocol (Vlahos et al., 2006; Chen et al., 2020). The experimental protocol in the same animals has been published in detail previously (Chen et al., 2020). Briefly, male Balb/c mice (7weeks, average body weight $20.3 \mathrm{~g}$ ) were housed in individually ventilated cages (3-5/cage) with Pura paper premium bedding and fed a commercially available pellet HFD (43\% energy from fat, $20 \mathrm{~kJ} / \mathrm{g}$, Cat\# SF03-20, Specialty Feeds, WA, Australia) for 16 weeks to induce obesity, with standard rodent chow as control (14\% energy from fat, $14 \mathrm{~kJ} / \mathrm{g}$, Gordon's Specialty Stockfeeds, NSW, Australia). From weeks 11-16, two sub-groups of mice in each dietary group were exposed to nicotine-containing [e-cig18, $18 \mathrm{mg} / \mathrm{ml}$ (regular strength), 50\% Propylene Glycol (PG)/50\%Vegetable Glycerin (VG), tobacco flavour and Vaper Empire, VIC] and nicotine-free e-vapour [e-cig0; 50\% Propylene Glycol (PG)/50\%Vegetable Glycerin (VG), tobacco flavour and Vaper Empire, VIC] in a $19 \mathrm{~L}$ chamber for $30 \mathrm{~min}$, twice daily for 6 weeks (Chen et al., 2020). The mice with sham exposure were exposed to room air in the identical chamber in the fume hood. Blood cotinine levels measured the following morning after the last e-vapour exposure in these mice were published previously to confirm nicotine exposure (Chen et al., 2020). This generated six experimental groups $(n=15)$, Chow + sham, Chow + e-cig18, Chow + e-cig0, HFD + sham, $\mathrm{HFD}+\mathrm{e}-\operatorname{cig} 18$ and HFD $+\mathrm{e}$-cig0. The $24 \mathrm{~h}$ caloric intake was measured once every 2 weeks by the weight difference of the pellets on the cage lid within $24 \mathrm{~h}$. Any residue pellets on bedding were also included. The average of caloric intake in the weeks 12, 14 and 16 weeks is reported here. At 15 weeks of feeding, an intraperitoneal glucose tolerance test was performed 
as previously described (Chen et al., 2014). After $5 \mathrm{~h}$ of fasting, the mice were challenged by glucose injection $(2 \mathrm{~g} / \mathrm{kg}$, ip) and blood glucose levels were measured at $0,15,30,60$ and $90 \mathrm{~min}$. The area under the curve (AUC) of the blood glucose curve was calculated for each mouse. At the endpoint, mice were fasted overnight. After the induction of deep anaesthesia ( $2 \%$ isoflurane), blood was collected via cardiac puncture, and the plasma was stored at $-20^{\circ} \mathrm{C}$ for further analysis. The retroperitoneal fat pad and liver were weighed, and livers were kept at $-80^{\circ} \mathrm{C}$.

\section{Bioassays}

Plasma, liver extracts and glycerol standards (Sigma-Aldrich, MO, United States) were incubated with triacylglycerol reagent (Roche Diagnostics, Basel, Switzerland) using an in-house assay as we previously described (Chen et al., 2018c). Plasma non-esterified free fatty acids (NEFA) were measured using a commercial NEFA kit (WAKO, Osaka, Japan) following the manufacturer's instructions (Chen et al., 2018c). Total liver lipid was measured by semi-quantitative oil red $\mathrm{O}(\mathrm{ORO})$ assay, as we previously described (Lo et al., 2011). Briefly, the ORO stock solution $0.25 \%$ (wt/vol) was freshly diluted in $10 \%$ dextran at 6:4 (ORO: dextran); then, $30-50 \mathrm{mg}$ liver was homogenised and incubated in the ORO working solution for $1 \mathrm{~h}$. After washing with $60 \%$ isopropanol to remove excess dye, the dye incorporated into lipid was extracted in $99 \%$ isopropanol. Absorbance at $520 \mathrm{~nm}$ was measured alongside an ORO standard curve.

\section{Real-Time PCR}

Gene expression was measured using our previously published protocol (Li et al., 2019) according to the MIQE guidelines (Bustin et al., 2009). Briefly, total mRNA was extracted from liver tissue with TriZol reagent following the manufacturer's instructions (Life Technologies, CA, United States) and used as a template to generate the first-strand CDNA using M-MLV Reverse Transcriptase, RNase H, Point Mutant Kit (Promega, WI, United States). Gene expression was quantified with manufacturer pre-optimised and validated $\mathrm{TaqMan}^{\circledR}$ primers and probes pre-optimised by the manufacture (Thermo Fisher, CA, United States) and standardised to 18s RNA (Li et al., 2019). The expression was calculated using $2^{-\Delta \Delta C t}$ and the Chow + sham group was assigned the calibrator against which all other results were expressed as fold changes.

\section{Western Blotting}

Western blotting was performed for fatty acid synthase (FASN). The liver was homogenised using cell lysis buffer (Hepes $20 \mathrm{~mm}$, EGTA $1 \mathrm{~mm}$, Mannitol $210 \mathrm{~mm}$ and Sucrose $70 \mathrm{~mm}$ ) for the whole protein with protease inhibitor (Thermo Fisher, United States, Cat. A32963, dissolve fresh in $50 \mathrm{ml}$ of lysis buffer). Protein samples $(40 \mu \mathrm{g})$ were separated on $4-15 \%$ Criterion TGX Precast Midi Protein Gel, 26 well (Bio-Rad, United States, Cat. 5671085) and then transferred to PVDF membranes with Trans-Blot Turbo Transfer System (Trans-blot Turbo Midi 0.2 $\mu \mathrm{m}$ PVDF Transfer pack; Bio-Rad, United States,
Cat. 1704157), which were blocked with non-fat milk powder and incubated with the primary antibodies FASN (1:2,000, Cat\#3180S, Cell Signalling) and $\beta$-action (1:1,000, Cat\#AHP2417, Bio-Rad) overnight and then secondary antibodies (1:5,000, goat anti-rabbit IgG horseradish peroxidase-conjugated secondary antibodies, Cat\#STAR124P, BIO-RAD) for $1 \mathrm{~h}$. Protein expression was detected by SuperSignal West Pico Chemiluminescent substrate (Thermo, MA, United States) by exposure of the membrane in Chemidoc MP (Bio-Rad, California, United States). Protein band density was measured using ImageJ software (National Institute of Health, Bethesda, Maryland, United States), with the results expressed as a ratio of the individual marker intensity relative to $\beta$-actin band intensity.

\section{Statistical Methods}

The results are expressed as mean \pm standard error of the mean (SEM) and analysed by two-way ANOVA followed by Fisher's least significant difference (LSD) post-hoc tests (GraphPad Prism 9, GraphPad, CA, United States). A conditional $t$-test was performed between the control (Chow + sham or HFD + sham) and interventional groups. $p<0.05$ was considered statistically significant.

\section{RESULTS}

\section{Adiposity and Lipid Profile}

All the groups had a similar body weight at baseline (Table 1). After 16 weeks of HFD consumption, the mice consuming a HFD were heavier than chow-fed mice receiving the same treatment $(p<0.05 \mathrm{HFD}+$ sham vs. Chow + sham group, Table 1). Nicotine-free e-vapour reduced body weight in chow-fed mice only $(p<0.05$, Chow $+\mathrm{e}-\mathrm{cig} 0$ vs. Chow + sham $)$. There was a trend of e-vapour exposure to reduce daily caloric intake independent of nicotine (Table 1), where only Chow $+\mathrm{e}-\mathrm{cig} 0$ group ate significantly less than the Chow + sham group $(p<0.05$ Table 1), consistent with their smaller body weight. Liver weight was not changed by HFD but was reduced by e-vapour exposure $(p<0.01$ overall e-vapour exposure effect; $p<0.05$ Chow + e-cig1 18 and Chow +e-cig0 vs. Chow + sham, HFD+sham vs. HFD + e-cig0, Table 1). HFD consumption nearly doubled the amount of retroperitoneal and epididymal fat masses (for both fat pads $p<0.01$, Chow + sham vs. HFD + sham, HFD $+\mathrm{e}-\operatorname{cig} 18$ vs. Chow +e-cig18; epididymal fat $p<0.01 \mathrm{HFD}+\mathrm{e}-\mathrm{cig} 0$ vs. Chow + e-cig0, Table 1). In chow-fed mice, e-vapour exposure also reduced retroperitoneal fat mass regardless of nicotine content ( $p<0.05$ vs. Chow-sham), with a similar trend in epididymal fat albeit no statistical significance. However, in HFD-fed mice, while retroperitoneal was reduced by exposure to nicotine-free e-vapour ( $p<0.05 \mathrm{HFD}+\mathrm{e}$-cig0 vs. HFD + sham), epididymal fat was not significantly changed by e-vapour exposure (Table 1).

High-fat diet consumption increased blood NEFA levels as expected $(p<0.05, \mathrm{HFD}+$ sham vs. Chow + sham group). There was an overall effect of e-vapour exposure to increase NEFA level in both chow and HFD-fed mice $(p<0.05)$; while significance 
TABLE 1 | Metabolic parameters.

\begin{tabular}{|c|c|c|c|c|c|c|c|}
\hline & Chow + sham & Chow +e-cig18 & Chow + e-cig0 & HFD + sham & HFD + e-cig18 & HFD + e-cig0 & Main effect $(p)$ \\
\hline Body weight at week 0 (g) & $20.3 \pm 0.26$ & $20.5 \pm 0.29$ & $20.2 \pm 0.20$ & $20.4 \pm 0.31$ & $20.2 \pm 0.43$ & $20.2 \pm 0.25$ & \\
\hline Body weight at week 16 (g) & $29.0 \pm 0.24$ & $27.4 \pm 0.6$ & $26.6 \pm 0.5^{*}$ & $30.3 \pm 0.60^{t}$ & $29.2 \pm 0.81$ & $28.2 \pm 0.46$ & $\begin{array}{l}\text { diet }<0.01 \\
\text { e-vapour }<0.01\end{array}$ \\
\hline Caloric intake (kJ/mouse/day) & $58.0 \pm 0.50$ & $52.7 \pm 3.31$ & $49.3 \pm 3.88^{*}$ & $54.6 \pm 2.21$ & $48.7 \pm 3.24$ & $49.7 \pm 2.39$ & \\
\hline Liver (g) & $1.26 \pm 0.04$ & $1.14 \pm 0.03^{*}$ & $1.12 \pm 0.03^{*}$ & $1.23 \pm 0.05$ & $1.20 \pm 0.03$ & $1.11 \pm 0.04^{\#}$ & e-vapour $<0.01$ \\
\hline Liver \% & $4.57 \pm 0.14$ & $4.37 \pm 0.14$ & $4.38 \pm 0.09$ & $4.32 \pm 0.14$ & $4.18 \pm 0.06$ & $4.02 \pm 0.10^{\ddagger}$ & $\operatorname{diet}<0.01$ \\
\hline Retroperitoneal white fat (g) & $0.19 \pm 0.02$ & $0.14 \pm 0.02^{t}$ & $0.13 \pm 0.01^{t}$ & $0.35 \pm 0.05^{* *}$ & $0.31 \pm 0.04^{\dagger \dagger}$ & $0.22 \pm 0.02^{\#}$ & $\begin{array}{l}\text { diet }<0.01 \\
\text { e-vapour }<0.05\end{array}$ \\
\hline Retroperitoneal white fat (\%) & $0.68 \pm 0.07$ & $0.45 \pm 0.08^{t}$ & $0.51 \pm 0.06^{t}$ & $1.17 \pm 0.16^{*}$ & $1.06 \pm 0.13^{\dagger+}$ & $0.81 \pm 0.06^{\#}$ & $\begin{array}{l}\text { diet }<0.01 \\
\text { e-vapour }=0.05\end{array}$ \\
\hline Epididymal white fat (g) & $0.50 \pm 0.03$ & $0.43 \pm 0.05$ & $0.40 \pm 0.02$ & $0.92 \pm 0.08^{* *}$ & $0.89 \pm 0.10^{\dagger \dagger}$ & $0.82 \pm 0.05^{\text {㧊 }}$ & $\operatorname{diet}<0.01$ \\
\hline Epididymal white fat (\%) & $1.82 \pm 0.11$ & $1.64 \pm 0.16$ & $1.55 \pm 0.07$ & $3.11 \pm 0.21^{* *}$ & $3.32 \pm 0.24^{+\dagger}$ & $2.98 \pm 0.16^{\ddagger \ddagger}$ & diet $<0.01$ \\
\hline AUC (mm·min) & $1,326 \pm 54$ & $1,206 \pm 70^{t}$ & $1,274 \pm 38$ & $1,481 \pm 62^{*}$ & $1,252 \pm 42^{\# \#}$ & $1,390 \pm 33$ & $\begin{array}{l}\text { diet }<0.05 \\
\text { e-vapour }<0.01\end{array}$ \\
\hline Plamsa NEFA (nmol)n & $3.71 \pm 0.38$ & $4.75 \pm 0.36^{*}$ & $4.65 \pm 0.23$ & $4.41 \pm 0.32^{*}$ & $4.60 \pm 0.11$ & $5.33 \pm 0.48^{\#}$ & e-vapour $<0.05$ \\
\hline Plasma tryglycerides $(\mathrm{mg} / \mathrm{ml})^{\eta}$ & $1.87 \pm 0.11$ & $2.05 \pm 0.14^{t}$ & $2.38 \pm 0.19^{t}$ & $2.75 \pm 0.28^{* *}$ & $2.09 \pm 0.22^{\#}$ & $2.43 \pm 0.18$ & \\
\hline Liver Oil red O (mg/g liver) $)^{n}$ & $45.6 \pm 9.25$ & $76.2 \pm 10.6^{*}$ & $79.1 \pm 14.4^{*}$ & $87.9 \pm 8.75^{*}$ & $82.5 \pm 7.92$ & $80.3 \pm 7.08$ & \\
\hline
\end{tabular}

The results are expressed as mean $\pm S E M, n=15$. Data were analysed by two-way ANOVA followed by Fisher's least significant difference (LSD) post-hoc tests. A conditional $t$-test for non-overlap of value distributions was performed between the control (Chow +sham or HFD + sham) and interventional groups. " $n=7-8$.

" $p<0.05 ; * *<0.01$ vs. Chow + Sham; ${ }^{t} p<0.05$ by conditional t-test vs. Chow + Sham.

"p<0.05; \#p<0.01 vs. HFD+Sham.

${ }^{t+} p<0.01$ vs. Chow + e-cig18.

${ }^{\ddagger} p<0.05 ;{ }^{+t} p<0.01$ vs. Chow - e-cig0.

AUC, area under the curve of blood glucose concentrations during an intraperitoneal glucose tolerance test; NEFA, non-esterified fatty acids.

was only seen between Chow + sham and Chow $+\mathrm{e}$-cig18 groups $(p<0.05)$, and between HFD + sham and HFD +e-cig0 groups $(p<0.05)$. HFD consumption also increased serum triglycerides levels $(p<0.01, \mathrm{HFD}+$ sham vs. Chow + sham group, Table 1), which was reduced by exposure to nicotine-containing e-vapour $(p<0.05, \mathrm{HFD}+\mathrm{e}$-cig18 vs. HFD + sham, Table 1). However, in chow-fed mice, blood triglycerides were increased by e-vapour exposure independent of nicotine $(p<0.05 \mathrm{Chow}+\mathrm{e}$-cig18 and Chow + e-cig0 vs. Chow + sham by conditional $t$-tests). Interestingly, the triglyceride concentration in the liver followed a similar pattern to triglycerides in the blood $(p<0.05$, Chow + sham vs. Chow +e-cig18, Chow +e-cig0 and HFD+sham, Table 1).

The de novo lipogenesis marker fatty acid synthase (FASN) was increased by HFD consumption and e-vapour exposure $(p<0.05, \mathrm{HFD}+$ sham vs. Chow + sham, HFD + e-cig18 vs. HFD + sham and Chow +e-cig18), with the effect more robust in the HFD $+\mathrm{e}-\operatorname{cig} 0$ group $(p<0.01$ vs. HFD + sham and Chow +e-cig0, Figure 1A). The lipolysis marker adipose triglyceride lipase (ATGL) was upregulated by nicotine-free e-vapour in chow-fed mice $(p<0.01$, Chow +e-cig0 vs. Chow + sham, Figure 1B) but was suppressed in HFD-fed mice with the same treatment $(p<0.01$, HFD + e-cig0 vs. HFD + sham, Figure 1B). The rate-limiting enzyme for lipid $\beta$-oxidation, carnitine palmitoyltransferase (CPT) 1a, was suppressed by HFD consumption $(p<0.01$, overall diet effect; $p<0.05$, HFD + sham vs. Chow + sham, Figure 1C), without any influence from e-vapour exposure.

\section{Glucose Metabolism}

During the glucose tolerance test, the glucose levels in the HFD + sham group were significantly higher than the
Chow + sham group at 30 and $60 \mathrm{~min}$ post-glucose injection $(p<0.05$, Figure $2 A)$. The HFD + cig18 group only had a higher glucose level than its chow-fed counterpart at $60 \mathrm{~min}(p<0.05$ vs. Chow + e-cig18), and the HFD + cig0 group had a higher glucose level than its chow-fed counterpart before glucose injection at Time $0(p<0.05$ vs. Chow + e-cig0, Figure 2A). Glucose intolerance in HFD + sham mice was confirmed by a greater AUC value for the glucose tolerance test $(p<0.05$, HFD + sham vs. Chow + sham group, Table 1), which was reduced by exposure to nicotine-containing e-vapour $(p<0.01$, HFD + e-cig 18 vs. HFD + sham). A similar effect was found in chow-fed mice $(p<0.05$ by conditional $t$-test, Chow $+\mathrm{e}$-cig 18 vs. Chow + sham).

We examined markers related to insulin sensitivity and glucose metabolism. The inflammatory marker TNF- $\alpha$ was increased by HFD consumption ( $p<0.05$ diet effect, Figure 2B), but no change was observed for IL-1 $\beta$ (Figure 2C), suggesting activation of macrophages but not the inflammasome. The total intrahepatic expression of collagen 1 (Colla) was also increased by HFD, as expected $(p<0.05$, Figure 2D), with significance observed between the Chow + e-cig0 and HFD + e-cig0 groups $(p<0.05)$. HFD suppressed the expression of the insulin sensing marker PPAR $\gamma(p<0.01$, Figure 2E), which was not affected by e-vapour exposure. There was an overall effect of diet to increase the intrahepatic expression of both Glut $2(p<0.01$, Figure 2F) and Glut $4(p<0.05$, Figure 2G), and e-vapour exposure increased Glut 2 expression $(p<0.05, \mathrm{HFD}+\mathrm{e}$-cig18 vs. HFD + sham, HFD +e-cig0 vs. HFD + sham). Overall, the changes in most of the above-mentioned markers were more marked in HFD + e-cig0 group, suggesting potential harm from the combination of HFD and exposure to nicotine-free e-vapour. Two gluconeogenesis markers, phosphoenolpyruvate 


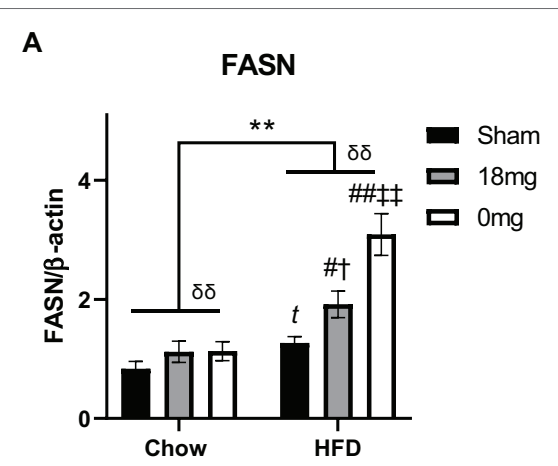

B

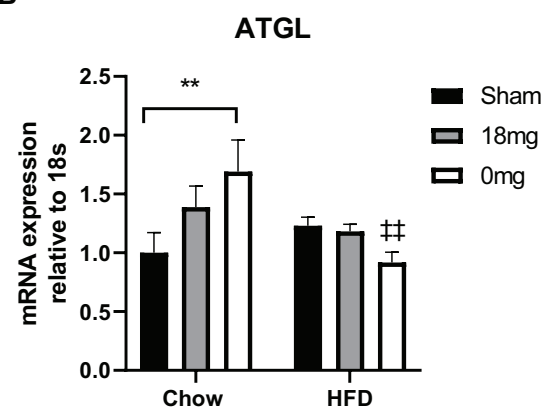

C

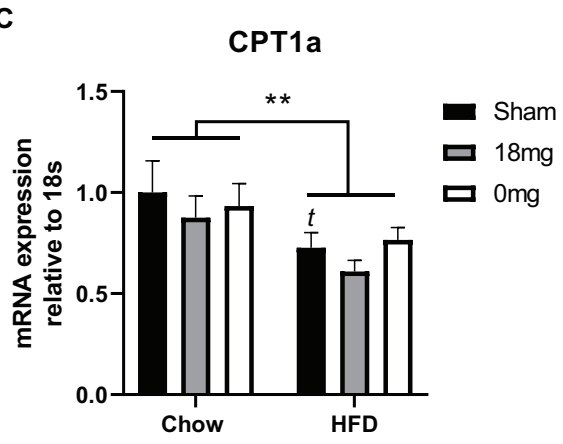

FIGURE 1 | Lipid metabolic markers in the liver. Protein level of FASN (A), mRNA expression of ATGL (B) and CPT1a (C) in mice fed a HFD with or without the exposure to e-vapour. The results are expressed as mean \pm SEM, $n=5-8$. Data were analysed by two-way ANOVA followed by Fisher's least significant difference (LSD) post-hoc tests. A conditional $t$-test for non-overlap of value distributions was performed between the control (Chow + sham or HFD + sham) and interventional groups. ${ }^{* *} p<0.01$ diet effect, ${ }^{8 \delta} p<0.01$ e-vapour exposure effect; $t p<0.05$ vs. Chow + Sham by conditional $t$-test, $\# p<0.05$, \# $p<0.01$ vs. HFD + Sham, ${ }^{\dagger} p<0.05$ vs. Chow + e-cig18mg and ${ }^{\ddagger \ddagger} p<0.01$ vs. Chow + e-cigO. ATGL, adipose triglyceride lipase; CPT, carnitine palmitoyltransferase; and FASN, fatty acid synthase.

carboxykinase (PEPCK) and Forkhead box protein O1 (FOXO1), were both suppressed by HFD consumption $(p<0.01$ overall diet effect, Figures $2 \mathbf{H}, \mathbf{I}$ ) with no influence from e-vapour exposure.

\section{DISCUSSION}

While there is no firm conclusion on whether e-cigarette use increases the risk of type 2 diabetes (Górna et al., 2020), our controlled animal model showed that sub-chronic exposure to a low dose of e-cigarette vapour does not impair glucose tolerance. On the contrary, in HFD-fed mice, nicotine-containing e-vapour improved glucose clearance, while nicotine-free e-vapour reduced retroperitoneal fat mass. However, while e-vapour exposure reduced fat mass in both chow and HFD-fed mice, there was a differentiated impact of e-vapour exposure on the lipid profile. In chow-fed mice, lipids in blood and liver were increased by e-vapour exposure independent of nicotine. In HFD-fed mice, blood NEFA and triglycerides were increased by nicotine-free e-vapour, while triglycerides were reduced by nicotine-containing e-vapour, with no impact on the liver triglycerides.

We used a HFD with a lower fat concentration (43\%) than others have used (>60\%; Espinoza-Derout et al., 2019; Hasan et al., 2019). However, energy from the dietary fat in this study is still higher than the average intake of adult Australian males, and $>22 \%$ higher than the upper limit of the accepted macronutrient distribution range (Grech et al., 2018). Different from our previous observations of significant weight gain in C57BL/6 mice using the same diet (Glastras et al., 2016; Chen et al., 2018b,c; Komalla et al., 2020), Balb/c mice had less than $5 \%$ weight gain, yet with nearly doubled retroperitoneal and epididymal fat masses. This is consistent with the strain susceptibility to HFD-induced obesity (Montgomery et al., 2013). In line with increased adiposity, blood levels of NEFA and triglycerides were also increased, suggesting the key role of fat mass in determining metabolic disorders. Therefore,
16 weeks of HFD consumption is sufficient to induce metabolic disorders. E-vapour exposure reduced retroperitoneal fat mass regardless of diet. This is somewhat similar to a previous study using cigarette smoke exposure with a similar nicotine level (Chen et al., 2007). Although one may consider this to be due to an effect of nicotine, retroperitoneal fat mass reduction in HFD-fed mice was more marked with nicotine-free e-vapour than the nicotine-containing one, with a similar trend observed in epididymal fat. This fat loss somewhat mirrors the suppressed caloric intake by e-vapours exposure which was independent of nicotine. This result suggests that both suppressed appetite and fat loss effect is most likely due to other chemicals in the e-vapour other than nicotine. Here, we cannot rule out the possibility of other additives [e.g. pyrazines (Clineschmidt et al., 1977)] in the e-vapour that may activate the reward pathway to suppress appetite, which requires future studies to identify.

There has been a dramatic increase in non-alcoholic fatty liver disease in recent years, due to increased intake of diets rich in simple carbohydrates and lipids that activate de novo lipid synthesis (Loomba et al., 2021). In this study, there was $>60 \%$ increase in liver lipid storage in HFD-fed mice $(\mathrm{HFD}+$ sham). Very likely contributing to increased lipid deposition, the enzyme for lipid synthesis FASN was upregulated while the regulator of lipid $\beta$-oxidation CPT-1a was downregulated, whereas the lipolytic enzyme ATGL was not changed. In addition, hepatic insulin resistance can reduce glucose conversion to glycogen and re-direct it to de novo lipid synthesis (Loomba et al., 2021). Indeed, the insulin sensing marker PPAR $\gamma$ was downregulated in $\mathrm{HFD}+$ sham mice, supporting this mechanism. HFD-fed mice had a significant increase in FASN from nicotine-containing e-vapour, and more so from nicotine-free e-vapour exposure. However, this change negatively correlated with triglyceride concentrations in the liver. As invoking the classical lipid metabolic regulators does not explain such changes from e-vapour exposure in HFD-fed mice, there may be an unknown mechanism that requires further investigation. Conversely, although nicotine has been 


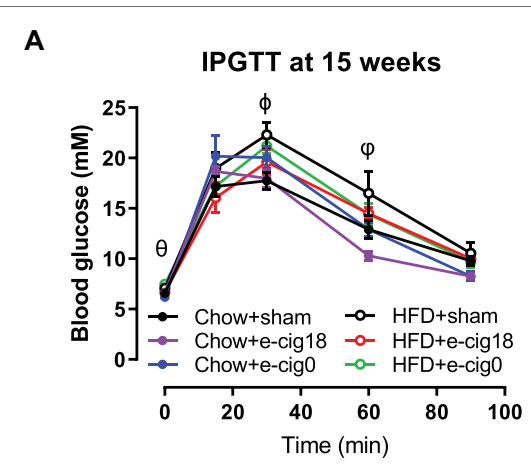

D

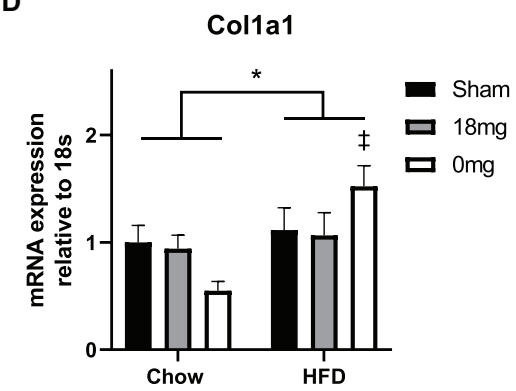

G

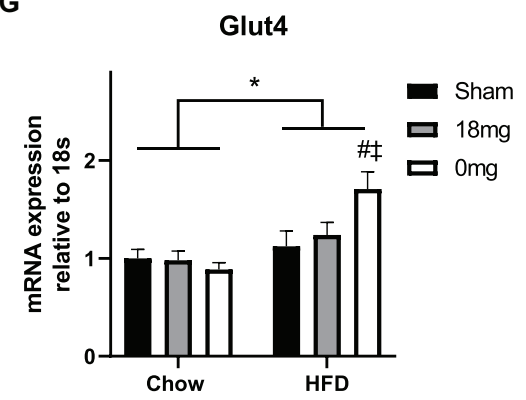

B

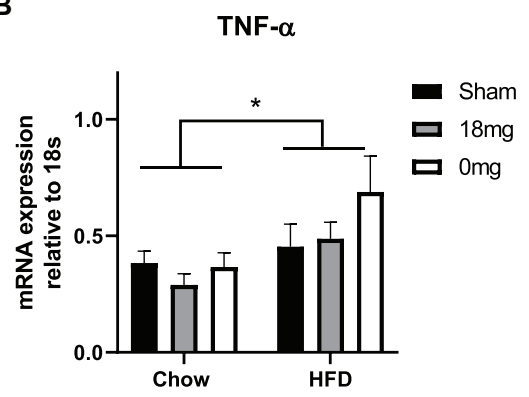

E

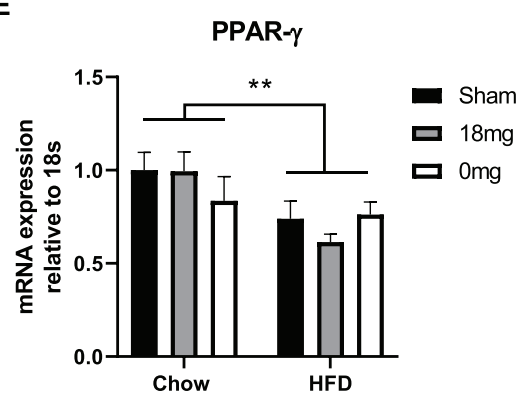

H

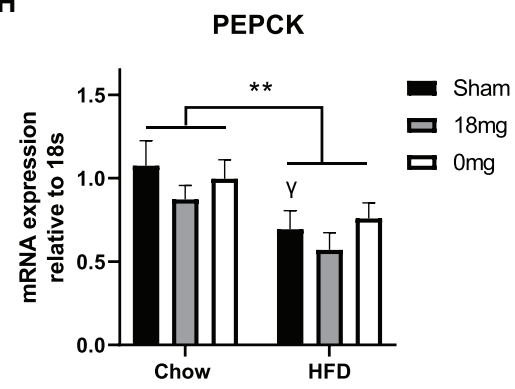

C

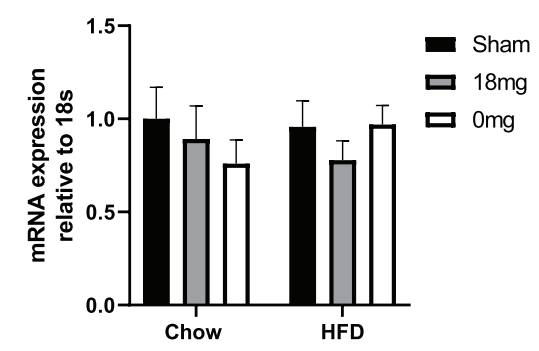

F

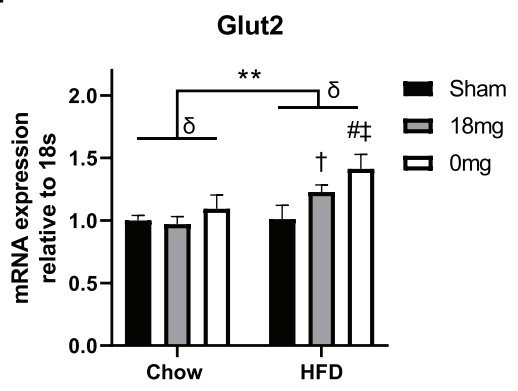

'

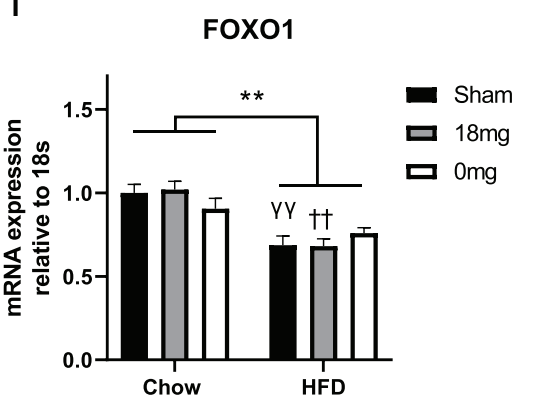

FIGURE 2 | Glucose level during intraperitoneal glucose tolerance test (IPGTT, A) and mRNA expression of markers of inflammation [TNF- $\alpha$ (B), IL-1 $\beta$ (C)], fibrosis (Col1a1, D), insulin sensing (PPAR $\gamma, \mathbf{E})$, glucose transporter [Glut2 (F), Glut4 (G)] and gluconeogenesis [PEPCK (H), FOXO1 (I)] in the liver. The results are expressed as mean \pm SEM, $n=5-8$. Data were analysed by two-way ANOVA followed by Fisher's least significant difference (LSD) post-hoc tests. ${ }^{\theta} p<0.05$ Chow + e-cig0 vs. HFD + e-cig0; ${ }^{\phi} p<0.01$ Chow + sham vs. HFD + sham; ${ }^{\oplus} p<0.05$ Chow + sham vs. HFD + sham; Chow + e-cig18 vs. HFD + e-cig18; ${ }^{\star} p<0.05$, ${ }^{* *} p<0.01$ diet effect, ${ }^{8} p<0.05$ e-vapour exposure effect; ${ }^{\gamma} p<0.05, r p<0.01$ vs. Chow + sham; and ${ }^{\#} p<0.05$ vs. HFD + Sham, ${ }^{\dagger} p<0.05 ;{ }^{\dagger \dagger} p<0.01$ vs. Chow + e-cig18 and ${ }^{\ddagger} p<0.05$ vs. Chow + e-cig0. Col1a1, collagen 1a1; FOXO1, Forkhead box protein O1; Glut, glucose transporter; PEPCK, phosphoenolpyruvate carboxykinase; and PPAR, Peroxisome proliferator-activated receptors.

shown to promote lipid accumulation in the liver (Sinha-Hikim et al., 2017; Chen et al., 2018d), here in chow-fed mice increased liver triglycerides seems to be independent of nicotine. It is more likely that the glycerine base of e-vapour can enter the bloodstream from the respiratory tract without restriction (Eissenberg and Maziak, 2020), which may be a contributor to increased blood and liver lipids in chow-fed mice exposed to e-vapour. A study in humans found that the addition of glycerine to a high-fat meal increased plasma free fatty acid level, possibly via chylomicron synthesis (Nicolaïew et al., 1995). While there is no direct evidence to support this speculation, a toxicity study did suggest intact humectant vegetable glycerine after heating (Li et al., 2021). Future studies can investigate this possibility. Nevertheless, lipolysis regulator ATGL was only upregulated by e-vapour exposure in chow-fed mice, which may also be an adaptive response to increased lipid content in the liver that is not successful in reversing the condition. This adaption can be impaired by long-term HFD consumption; thus, ATGL was not increased in HFD-fed mice with the same e-vapour exposure. In summary, e-vapour exposure may increase the risk of fatty liver disorder regardless of nicotine, which does not exacerbate lipid deposition due to HFD consumption.

The liver regulates peripheral glucose metabolism. Local inflammation due to residential macrophages plays a key role in liver insulin resistance due to HFD diet consumption (Lo et al., 2011), exemplified by increased TNF- $\alpha$ and synchronously reduced PPAR $\gamma$ expression in HFD-fed mice 
in this study. It is believed that nicotine is anti-inflammatory (Kalra et al., 2004; Cohen et al., 2007), which was not reflected in the HFD+e-cig 18 and HFD+e-cig0 mice, potentially due to the low dose of nicotine in this study. Liver fibrosis is directly linked to local inflammation and ectopic lipid accumulation. Here, collagen was increased only by HFD consumption and not by e-vapour exposure.

Forkhead box protein $\mathrm{O} 1$ and its promoter PEPCK regulate gluconeogenesis, raising blood glucose levels. They are upregulated during dietary obesity and type 2 diabetes, resulting in hyperglycaemia and glucose intolerance (Nakae et al., 2001; Seth et al., 2013). As a result, suppressing FOXO1 signalling can protect mice from HFD-induced insulin resistance by increasing insulin sensing regulator PPAR $\gamma$ (Kim et al., 2009). However, in this model, both PEPCK and FOXO1 were suppressed by HFD. We hypothesise it may be another adaptative response. Our results of improved glucose tolerance in the Chow $+\mathrm{e}$-cig 18 and HFD+e-cig18 mice are similar to a previous study using a low level e-vapour exposure and chronic nicotine administration (Thompson et al., 1990; Vu et al., 2014; Orimoloye et al., 2019). Moreover, PEPCK has also been shown to be suppressed by chronic nicotine administration (Vu et al., 2014). Previously, cold e-fluid which has a different composition to e-cigarette vapour generated by an e-cigarette device, was intraperitoneally injected to study glycaemic control (El Golli et al., 2016). To our knowledge, no other study has used a setting similar to ours (i.e. using e-vapour with/without nicotine in combination with a HFD), with which to directly compare the finding. Nevertheless, the improved glucose tolerance by nicotinecontaining e-vapour is similar to the effect of tobacco cigarette smoke at a does equivalent to light smokers (Chen et al., 2007). The comparison with the groups exposed to nicotinefree e-vapour ( $\mathrm{HFD}+\mathrm{e}$-cig0) further suggests that the improvement of glucose tolerance is most likely to be nicotine driven, although our studies have not defined the mechanistic basis for this improvement.

We demonstrated increased glucose transporters in HFD-fed mice with e-vapour exposure, especially nicotine-free e-vapour. With downregulated gluconeogenesis markers, this may partially explain why glucose tolerance was improved in mice exposed to e-vapour. However, the glycaemic control effect was still more potent with nicotine-containing e-vapour, consistent with the effect of pure nicotine to increase glucose uptake in the literature (Liu et al., 2003; Vu et al., 2014). However, glucose may not be converted to triglycerides for storage. How the additional glucose was disposed of needs further investigation.

We need to acknowledge several limitations in this study. Firstly, we only used a low dose of e-vapour exposure, which is equivalent to light smokers. Higher doses of e-vapour may introduce more toxic chemicals, potentially having different effects on lipid and glucose metabolism. Secondly, we exposed the mice to e-vapour for 6 weeks, which is a relatively short duration compared with human usage. Some improvement in glucose or adiposity may be a 'honeymoon' adaptative effect, considering the unfavourable changes in FASN, PPAR $\gamma$ and glucose transporters in the liver. Thirdly, we used Balb/c mice, which is not an obese-prone strain and the ability of the liver to uptake lipids is also lower than other strains (Montgomery et al., 2013). As such, the adverse or beneficial effects on nutrient metabolism of e-vapour exposure need to be interpreted with caution. Lastly, this short communication only reports the initial finding in the males to raise the attention of the field in the setting of HFD combined by e-vaping. We would expect similar changes in the females, as there seems to be no sex difference in the metabolic disturbance in response to environmental insults (Nguyen et al., 2015; Saad et al., 2018). Future studies can follow up on the mechanisms, such as the involvement of the gut microbiome, and confirm the changes in the females.

\section{CONCLUSION}

In this study, e-vapour exposure reduced fat mass in both chow and HFD-fed mice but showed a differential effect on lipid profile with different diets. In mice fed a balanced diet, it tends to increase lipid levels in both blood and liver. However, in mice fed a diet rich in lipid and simple carbohydrates, it tends to reduce triglycerides but increase NEFA levels in the blood. In addition, low-dose nicotine inhalation seems to benefit glucose tolerance in HFD-fed mice; however, this should not be translated to humans who have more lifestyle confounders than laboratory animals.

\section{DATA AVAILABILITY STATEMENT}

The original contributions presented in the study are included in the article/supplementary material, and further inquiries can be directed to the corresponding author.

\section{ETHICS STATEMENT}

The animal study was reviewed and approved by the Animal Ethics and Care Committee at Northern Sydney Local Health District.

\section{AUTHOR CONTRIBUTIONS}

$\mathrm{HC}$, SS, and BO designed the study. HC, GL, YC, and HZ performed the experiments, collected the data, and analysed the results. HC prepared the figures and tables and wrote the first draft. All authors contributed to the writing of the manuscript, reviewed the final manuscript and have read and agreed to the published version of the manuscript.

\section{FUNDING}

This work was supported by a project grant from Australian National Health and Medical Research Council (AP1158186) and University of Technology Sydney. 


\section{REFERENCES}

Alpert, H. R., Agaku, I. T., and Connolly, G. N. (2015). A study of pyrazines in cigarettes and how additives might be used to enhance tobacco addiction. Tob. Control. 25, 444-450. doi: 10.1136/tobaccocontrol-2014-051943

Bustin, S. A., Benes, V., Garson, J. A., Hellemans, J., Huggett, J., Kubista, M., et al. (2009). The MIQE guidelines: minimum information for publication of quantitative real-time PCR experiments. Clin. Chem. 55, 611-622. doi: 10.1373/clinchem.2008.112797

Chen, H., Hansen, M. J., Jones, J. E., Vlahos, R., Anderson, G., and Morris, M. J. (2007). Detrimental metabolic effects of combining long term cigarette smoke exposure and high-fat diet in mice. Am. J. Physiol. Endocrinol. Metab. 293, E1564-E1571. doi: 10.1152/ajpendo.00442.2007

Chen, H., Li, G., Chan, Y. L., Chapman, D. G., Sukjamnong, S., Nguyen, T., et al. (2018a). Maternal e-cigarette exposure in mice alters DNA methylation and lung cytokine expression in offspring. Am. J. Respir. Cell Mol. Biol. 58, 366-377. doi: 10.1165/rcmb.2017-0206RC

Chen, H., Ng, J. P. M., Bishop, D. P., Milthorpe, B. K., and Valenzuela, S. M. (2018b). Gold nanoparticles as cell regulators: beneficial effects of gold nanoparticles on the metabolic profile of mice with pre-existing obesity. J. Nanobiotechnol. 16:88. doi: 10.1186/s12951-018-0414-6

Chen, H., Ng, J. P. M., Tan, Y., Mcgrath, K., Bishop, D. P., Oliver, B., et al. (2018c). Gold nanoparticles improve metabolic profile of mice fed a highfat diet. J. Nanobiotechnol. 16:11. doi: 10.1186/s12951-018-0338-1

Chen, X., Owoseni, E., Salamat, J., Cederbaum, A. I., and Lu, Y. (2018d). Nicotine enhances alcoholic fatty liver in mice: role of CYP2A5. Arch. Biochem. Biophys. 657, 65-73. doi: 10.1016/j.abb.2018.09.012

Chen, H., Simar, D., Pegg, K., Saad, S., Palmer, C., and Morris, M. (2014). Exendin- 4 is effective against metabolic disorders induced by intrauterine and postnatal overnutrition in rodents. Diabetologia 57, 614-622. doi: 10.1007/ s00125-013-3132-5

Chen, H., Wang, B., Li, G., Steele, J. R., Stayte, S., Vissel, B., et al. (2020). Brain health is independently impaired by e-vaping and high-fat diet. Brain Behav. Immun. 92, 57-66. doi: 10.1016/j.bbi.2020.11.028

Clineschmidt, B. V., Hanson, H. M., Pflueger, A. B., and Mcguffin, J. C. (1977). Anorexigenic and ancillary actions of MK-212 (6-chloro-2-[1-piperazinyl]pyrazine; CPP). Psychopharmacology 55, 27-33. doi: 10.1007/BF00432813

Cohen, L., Xueping, E., Tarsi, J., Ramkumar, T., Horiuchi, T. K., Cochran, R., et al. (2007). Epithelial cell proliferation contributes to airway remodeling in severe asthma. Am. J. Respir. Crit. Care Med. 176, 138-145. doi: 10.1164/ rccm.200607-1062OC

Eissenberg, T., and Maziak, W. (2020). Are electronic cigarette users at risk for lipid-mediated lung injury? Am. J. Respir. Crit. Care Med. 201, 1012-1013. doi: 10.1164/rccm.201910-2082LE

El Golli, N., Dkhili, H., Dallagi, Y., Rahali, D., Lasram, M., Bini-Dhouib, I., et al. (2016). Comparison between electronic cigarette refill liquid and nicotine on metabolic parameters in rats. Life Sci. 146, 131-138. doi: 10.1016/j. lfs.2015.12.049

Espinoza-Derout, J., Shao, X. M., Bankole, E., Hasan, K. M., Mtume, N., Liu, Y., et al. (2019). Hepatic DNA damage induced by electronic cigarette exposure is associated with the modulation of NAD+/PARP1/SIRT1 axis. Front. Endocrinol. 10:320. doi: 10.3389/fendo.2019.00320

Glastras, S. J., Chen, H., Teh, R., Mcgrath, R. T., Chen, J., Pollock, C. A., et al. (2016). Mouse models of diabetes, obesity and related kidney disease. PLoS One 11:e0162131. doi: 10.1371/journal.pone.0162131

Górna, I., Napierala, M., and Florek, E. (2020). Electronic cigarette use and metabolic syndrome development: a critical review. Toxics 8:105. doi: 10.3390/ toxics 8040105

Grech, A., Rangan, A., and Allman-Farinelli, M. (2018). Macronutrient composition of the Australian population's diet; trends from three National Nutrition Surveys 1983, 1995 and 2012. Nutrients 10:1045. doi: 10.3390/nu10081045

Hasan, K. M., Friedman, T. C., Shao, X., Parveen, M., Sims, C., Lee, D. L., et al. (2019). E-cigarettes and western diet: important metabolic risk factors for hepatic diseases. Hepatology 69, 2442-2454. doi: 10.1002/hep.30512

Kalra, R., Singh, S. P., Pena-Philippides, J. C., Langley, R. J., Razani-Boroujerdi, S., and Sopori, M. L. (2004). Immunosuppressive and anti-inflammatory effects of nicotine administered by patch in an animal model. Clin. Vaccine Immunol. 11, 563-568. doi: 10.1128/CDLI.11.3.563-568.2004
Kim, T., Choi, H., Kang, J., and Kim, J. (2020). Association between electronic cigarette use and metabolic syndrome in the Korean general population: a nationwide population-based study. PLoS One 15:e0244720. doi: 10.1371/ journal.pone. 0244720

Kim, J. J., Li, P., Huntley, J., Chang, J. P., Arden, K. C., and Olefsky, J. M. (2009). FoxO1 haploinsufficiency protects against high-fat diet-induced insulin resistance with enhanced peroxisome proliferator-activated receptor $\gamma$ activation in adipose tissue. Diabetes 58, 1275-1282. doi: 10.2337/db08-1001

Komalla, V., Sheikholeslami, B., Li, G., Bokshi, B., Chan, Y. L., Ung, A., et al. (2020). Impact of a cargo-less liposomal formulation on dietary obesityrelated metabolic disorders in mice. Int. J. Mol. Sci. 21:7640. doi: 10.3390/ ijms 21207640

Lanza, H. I., Pittman, P., and Batshoun, J. (2017). Obesity and cigarette smoking: extending the link to e-cigarette/vaping use. Am. J. Health Behav. 41, 338-347. doi: $10.5993 / \mathrm{AJHB} .41 .3 .13$

Layden, J. E., Ghinai, I., Pray, I., Kimball, A., Layer, M., Tenforde, M., et al. (2019). Pulmonary illness related to e-cigarette use in Illinois and Wisconsinpreliminary report. N. Engl. J. Med. 382, 903-916. doi: 10.1056/NEJMoa1911614

Li, Y., Burns, A. E., Tran, L. N., Abellar, K. A., Poindexter, M., Li, X., et al. (2021). Impact of e-liquid composition, coil temperature, and puff topography on the aerosol chemistry of electronic cigarettes. Chem. Res. Toxicol. 34, 1640-1654. doi: 10.1021/acs.chemrestox.1c00070

Li, G., Chan, Y. L., Sukjamnong, S., Anwer, A. G., Vindin, H., Padula, M., et al. (2019). A mitochondrial specific antioxidant reverses metabolic dysfunction and fatty liver induced by maternal cigarette smoke in mice. Nutrients 11:1669. doi: 10.3390/nu11071669

Liu, R. H., Mizuta, M., and Matsukura, S. (2003). Long-term oral nicotine administration reduces insulin resistance in obese rats. Eur. J. Pharmacol. 458, 227-234. doi: 10.1016/S0014-2999(02)02726-7

Lo, L., Mclennan, S. V., Williams, P. F., Bonner, J., Chowdhury, S., Mccaughan, G. W., et al. (2011). Diabetes is a progression factor for hepatic fibrosis in a high fat fed mouse obesity model of non-alcoholic steatohepatitis. J. Hepatol. 55, 435-444. doi: 10.1016/j.jhep.2010.10.039

Loomba, R., Friedman, S. L., and Shulman, G. I. (2021). Mechanisms and disease consequences of nonalcoholic fatty liver disease. Cell 184, 2537-2564. doi: 10.1016/j.cell.2021.04.015

Lumeng, C. N., Delproposto, J. B., Westcott, D. J., and Saltiel, A. R. (2008). Phenotypic switching of adipose tissue macrophages with obesity is generated by spatiotemporal differences in macrophage subtypes. Diabetes 57, 3239-3246. doi: $10.2337 / \mathrm{db} 08-0872$

Montgomery, M. K., Hallahan, N. L., Brown, S. H., Liu, M., Mitchell, T. W., Cooney, G. J., et al. (2013). Mouse strain-dependent variation in obesity and glucose homeostasis in response to high-fat feeding. Diabetologia 56, 1129-1139. doi: 10.1007/s00125-013-2846-8

Nakae, J., Kitamura, T., Silver, D. L., and Accili, D. (2001). The forkhead transcription factor Foxol (Fkhr) confers insulin sensitivity onto glucose-6phosphatase expression. J. Clin. Invest. 108, 1359-1367. doi: 10.1172/ JCI200112876

Nguyen, L. T., Stangenberg, S., Chen, H., Al-Odat, I., Chan, Y. L., Gosnell, M. E., et al. (2015). L-carnitine reverses maternal cigarette smoke exposure-induced renal oxidative stress and mitochondrial dysfunction in mouse offspring. Am. J. Physiol. Renal Physiol. 308, F689-F696. doi: 10.1152/ajprenal.00417.2014

Nicolaïew, N., Cavallero, E., Gandjini, H., Dolé, E., Koziet, J., Gambert, P., et al. (1995). Effect of acute glycerol administration with or without a mixed meal in humans. Ann. Nutr. Metab. 39, 71-84. doi: 10.1159/000177846

Oh, S. W., Yoon, Y. S., Lee, E. S., Kim, W. K., Park, C., Lee, S., et al. (2005). Association between cigarette smoking and metabolic syndrome: the Korea National Health and Nutrition Examination Survey. Diabetes Care 28, 2064-2066. doi: 10.2337/diacare.28.8.2064

Orimoloye, O. A., Uddin, S. M. I., Chen, L.-C., Osei, A. D., Mirbolouk, M., Malovichko, M. V., et al. (2019). Electronic cigarettes and insulin resistance in animals and humans: results of a controlled animal study and the National Health and Nutrition Examination Survey (NHANES 2013-2016). PLoS One 14:e0226744. doi: 10.1371/journal.pone.0226744

Pisinger, C., and Døssing, M. (2014). A systematic review of health effects of electronic cigarettes. Prev. Med. 69, 248-260. doi: 10.1016/j.ypmed.2014.10.009

Saad, S., Al-Odat, I., Chan, Y. L., Mcgrath, K., Pollock, C. A., Oliver, B. G., et al. (2018). Maternal L-carnitine supplementation improves glucose and 
Chen et al.

E-vaping and HFD on Metabolism

lipid profiles in female offspring of dams exposed to cigarette smoke. Chin. Exp. Pharmacol. Physiol. 45, 694-703. do: 10.1111/1440-1681.12921

Seth, R. K., Kumar, A., Dos, S., Kadiiska, M. B., Michelotti, G., Diehl, A. M., et al. (2013). Environmental toxin-linked nonalcoholic steatohepatitis and hepatic metabolic reprogramming in obese mice. Toxicol. Sci. 134, 291-303. dor: $10.1093 /$ toxsci/kft104

Sinha-Hikim, A. P., Sinha-Hikim, I., and Friedman, T. C. (2017). Connection of nicotine to diet-induced obesity and non-alcoholic fatty liver disease: cellular and mechanistic insights. Front. Endocrinol. 8:23. doe: 10.3389/fendo.2017.00023

Thompson, S. G., Stone, R., Nanchahal, K., and Wald, N. J. (1990). Relation of urinary cotinine concentrations to cigarette smoking and to exposure to other people's smoke. Thorax 45, 356-361. doe: 10.1136/thx.45.5.356

Vlahos, R., Bozinovski, S., Jones, J. E., Powell, J., Gros, J., Lilia, A., et al. (2006). Differential protease, innate immunity and NF kappa B induction profiles during lung inflammation induced by sub-chronic cigarette smoke exposure in mice. Am. J. Physiol. Lung Cell. Mol. Physiol. 290, L931-L945. do: 10.1152/ajplung.00201.2005

Nu, C. U., Siddiqui, J. A., Wadensweiler, P., Gayen, J. R., Avolio, E., Bandyopadhyay, G. K., et al. (2014). Nicotinic acetylcholine receptors in glucose homeostasis: the acute hyperglycemic and chronic insulin-sensitive effects of nicotine suggest dual opposing roles of the receptors in male mice. Endocrinology 155, 3793-3805. doa: 10.1210/en.2014-1320
Weitzman, M., Cook, S., Auinger, P., Florin, T. A., Daniels, S., Nguyen, M., et al. (2005). Tobacco smoke exposure is associated with the metabolic syndrome in adolescents. Circulation 112, 862-869. doa: 10.1161/ CIRCULATIONAHA.104.520650

Conflict of Interest: The authors declare that the research was conducted in the absence of any commercial or financial relationships that could be construed as a potential conflict of interest.

Publisher's Note: All claims expressed in this article are solely those of the authors and do not necessarily represent those of their affiliated organizations, or those of the publisher, the editors and the reviewers. Any product that may be evaluated in this article, or claim that may be made by its manufacturer, is not guaranteed or endorsed by the publisher.

Copyright (C) 2021 Chen, Li, Chan, Chang, Correl, Pollock, Salad and Oliver. This is an open-access article distributed under the terms of the Creative Commons Attribution License (CC BY). The use, distribution or reproduction in other forums is permitted, provided the original authors) and the copyright owner (s) are credited and that the original publication in this journal is cited, in accordance with accepted academic practice. No use, distribution or reproduction is permitted which does not comply with these terms.

Frontiers in Physiology | www.frontiersin.org

9

November 2021 | Volume 12 | Article 755124 